\section{RATSCA: A FORTRAN program to analyze rating scale data on a minicomputer}

\author{
HOWARD LEE and BARRY L. BERSON \\ Integrated Sciences Corporation, Santa Monica, California 90401
}

Description. Rating scale procedures are perhaps the most widely used subjective judgment technique in psychological measurement. The use of a rating scale encompasses the rating of stimuli or persons $(\geqslant 2)$ on certain traits $(\geqslant 2)$ by raters or judges $(\geqslant 2)$. An example would be the rating of five math professors on research ability and teaching prowess by 30 college students. Rating scales are applicable in a wide variety of situations. They can be used to craluate individuals, their reactions, and their products (Guilford, 1954).

In comparison to paired-comparisons and rank-order techniques, rating scales have compared quite favorably in many respects. Researchers have found the rating scale methodology to have the following advantages over the other two methods: (1) requires less time for administration; (2) has wider range of applicability; (3) can be used with psychologically naive raters who have had a minimum of training; and (4) can be used with a larger number of stimuli ( $\geqslant 25$ ).

However, in using rating scales, the user assumes that the human observer is capable of precision and objectivity. Furthermore, the ratings obtained from each judge are to be taken as accurate descriptions of the object rated. As sound as the judgments may be, ratings could be contaminated by the raters' personal biases. These biases are identified as (1) leniency error; (2) rater-trait interaction error, and (3) halo effect. Hence, the rating of a person $I$ on a trait $J$ by a rater $K$ is equal to the "true value" of person I on trait $J$ plus an error factor. This error factor.can be dissected into the three types of error presented above and a residual error. To estimate the error contributions to the ratings, the program abstracted here will perform three two-way analyses of variance on the rating data. Through these analy ses, an estimate of the true variation may be obtained. With this estimate, the reliability of ratings is calculated using Ebel's formula (1951). Adjusted ratings are derived by subtracting the values of the three errors calculated in the analyses of variance from the raw rating data. These ratings represent the rater's true rating of the person or stimulus being evaluated.

ine primary contribution of this program is its usability on minicomputers that have limited memory. The authors have used several programming "tricks" in this program to save computer core. Since it is written in FORTRAN IV, it is compatible to computer systems that possess ANSI FORTRAN compilers.

Capacity. The maximum number of stimuli (persons) is 40 , traits is 15 , and raters (judges) is 15 . However, these specifications may be changed by the user to fit the amount of memory available. This program has been developed for the minicomputer with limited memory. It can be adapted easily to larger machines.

Limitations. The minimum number of stimuli, traits, and raters is two.

Computer. Varian 73, $20 \mathrm{~K}$.

Program language. FORTRAN IV. Includes a subroutine PRBF developed by Veldman (1967).

Input. Input for this program is through a Teletype. The user is requested by the program to input the number of traits, ratees (stimuli), and raters (judges). The user nust then input the rating scale datat under l-type format.

Output. The program will output the three analysis of variance summary tables The first ANOVA summary contains the analysis of raters with traits with ratees held constant. The second ANOVA summary contains the analysis of raters with ratees and traits held constant. The third ANOVA summary contains the analysis of ratees and traits with raters held constant. The reliability of the ratings for a single rater and for all raters are outputted. The Spearman-Brown reliability coefficient is also given. Also outputted are the rater errors and the adjusted ratings.

Availability. The source listings, sample problem, program description, results interpretation, and scaling algorithm can be obtained from Howard Lee or Barry L. Berson, Integrated Sciences Corporation, 1532 Third Street, Suite 201, Santa Monica, California 90401, at no cost.

\section{REFERENCES}

Ebel, $R$. L. Estimation of the reliability of ratings. Psychometrika, 1951, 16, 407-424.

Guilford, J. P. Psychometric methods. New York: McGraw-Hill Book Company, 1954.

Veldman, Donald Fortran programming for the behavioral sciences. New York: Holt, Rinehart, and Winston, 1967.

\section{RAKPRO: A FORTRAN program to find scale values for rank order and paired comparison data on a mini-computer

\author{
BARRY L. BERSON and HOWARD LEE \\ Integrated Sciences Corporation \\ Santa Monica, California 90401
}

The method of rank order is one of the most popular and most practical of the psychometric methods. Its appeal has consisted largely in its ability to handle from small to relatively large numbers of stimuli (up to 40), and also in its wide range of applicability. This method forces the judges to make the maximum number of discriminations and thus provides as much discriminatory information as it is possible to obtain from them. Stimuli that have been ranked by a number of judges can be pooled to form a group rank ordering. From this group ordering, interval scale values can be derived.

In comparison to other psychometric methods, rank ordering is easier for judges to use than the method of paired comparisons and consumes much less time. Ratings are usually preferred over ranking when the number of stimuli is very large $(>40)$. However, when the number of stimuli is smaller than 40 , rankings are usually recommended because they are less prone to judges' biases.

The computer program abstracted here utilizes Guilford's (1954) composite standard method to derive scale values for the rank order data. The basic assumption for this approach is that each stimulus is judged in comparison with the group as a whole. The whole group then becomes a composite standard (CS) with which every stimulus is compared. Linear scale values are derived from the proportions of judgments given to every stimulus as compared with the CS.

Interval scale values are derived from the agreement between judges on how each stimuli should be ranked. The scale ranges from 0 to 1 . A scale value of 0 represents perfect agreement between judges that stimulus $J$ should be ranked last and where a scale value of 1 represents agreement between judges that Stimulus I should be ranked first. 